Geology, Geophysics \& Environment • 2012 • Vol. 38 • No. 4 • 395-410

http://dx.doi.org/10.7494/geol.2012.38.4.395

\title{
APPLICATION OF ELECTROMAGNETIC FIELD MODELLING IN GPR INVESTIGATIONS OF AN HISTORIC TENEMENT
}

\author{
Klaudia CZAJA \\ AGH University of Science and Technology, Faculty of Geology, \\ Geophysics and Environmental Protection, Department of Geophysics; \\ al. Mickiewicza 30, 30-059 Krakow, Poland; \\ e-mail:kczaja@agh.edu.pl
}

\begin{abstract}
The article presents the results of Ground Penetrating Radar (GPR) measurements carried out in a historic tenement in Krakow. The aim of this study was to check if there is a basement under the apartment's floor. In order to verify the results, modelling attempt of electromagnetic field evolution in the medium was undertaken. The numerical simulation of GPR impulse passage in the medium uses the finite-difference time-domain method (FDTD). Synthetic radargrams obtained as a result of the modelling were compared with measurements processed radargrams.
\end{abstract}

Key words: Ground Penetrating Radar, electromagnetic field modelling, historical buildings

\section{INTRODUCTION}

Ground Penetrating Radar (GPR) belongs to non-invasive geophysical methods, which use an artificially induced electromagnetic field as the way for inspection. GPR has many different applications such as: recognition of shallow geological structure, measuring snow depth, imaging concrete structure to locate the embedded objects (Saarenketo 2000, Annan 2002). Moreover GPR is used for geotechnical inspections of pavement and subpavement layers, in bridges inspections (Benedetto et al. 2007, 2012a, b) and in hydrogeology for estimation of volumetric water content and determination of water table level (Grote et al. 2003). GPR is applied also in investigations of historical buildings.

It is possible to obtain good results only in case of differences in dielectric constant between the adjacent geological layers in the area studied. For that reason this method is used to locate empty spaces and moist zones. Sometimes the obtained result leads to ambiguous interpretation despite the suitable measuring methodology and the proper GPR data 
processing. In such cases the computer modelling of the electromagnetic field in the medium is helpful. Modelling is applied also to select the most appropriate measurement parameters, when electric properties and boundary conditions in the medium are defined.

GPR allows for location of the anomaly zones caused by the occurrences of different objects and the structures and also for determination of distances to these objects and their sizes by the use of the electromagnetic waves. This is possible only when the object being searched for differs significantly from the surrounding medium with the value of the permittivity (Daniels 2004).

Permittivity and the electromagnetic wave attenuation are the most important parameters of the medium for the ground penetrating radar method. It is essential to define their influence on the resultant electromagnetic field because not only depth range but also spatial resolution of GPR is dependent on these parameters. The most important aspect of the GPR method is the phenomenon of electromagnetic wave reflection at the boundary between two media with differing electric properties (Karczewski 2007).

GPR reflection profiling entails simultaneous movement of both the transmitting and receiving antennas along the profile line. Such kind of measurement makes it possible to receive the information about a subsurface structure and in particular about the location of inhomogeneities and discontinuities of lithological layers.

Reflection profiling can be conducted in single profiling variant, 3D profiling (in the network of parallel profiles) or multichannel profiling. Echogram - time section where the vertical axis is the time axis and the horizontal axis corresponds to distance - is the result of profiling. After "time to depth" conversion, where average electromagnetic wave velocity is assumed, the vertical axis represents the depth of profile.

\section{OBJECTIVE AND CASE STUDY DESCRIPTION}

The aim of the measurements performed in a Krakow residence was to check if there was an empty space under the floor of an apartment. In the tenement the gate to the courtyard had been built over and this area was adapted for a flat. There were indications that a basement might be located below but no entrance to it could be found, and therefore direct verification of this hypothesis was impossible (Czaja 2010). In the other part of this tenement, there is basement with the entrance leading to it, and the foundations are made of bricks. The thickness of the vault of this cellar is between 1 and 2 meters and arch-shaped. The tenement was built in the end of $17^{\text {th }}$ century.

The measurements were performed using the GPR equipment from the Swedish company Mala Geoscience RAMAC / GPR. Due to the required depth range and resolution shielded, ground-coupled antennas with frequencies of $500 \mathrm{MHz}$ and $800 \mathrm{MHz}$ were used. During measurements the six profiles were traced and two of them will be discussed here. The first one is the P3 profile, which runs in a room parallel to the window. The second is profile $\mathrm{P} 4$, which runs from a window to the apartment entrance and is perpendicular to the profile P3 (Fig. 1). 

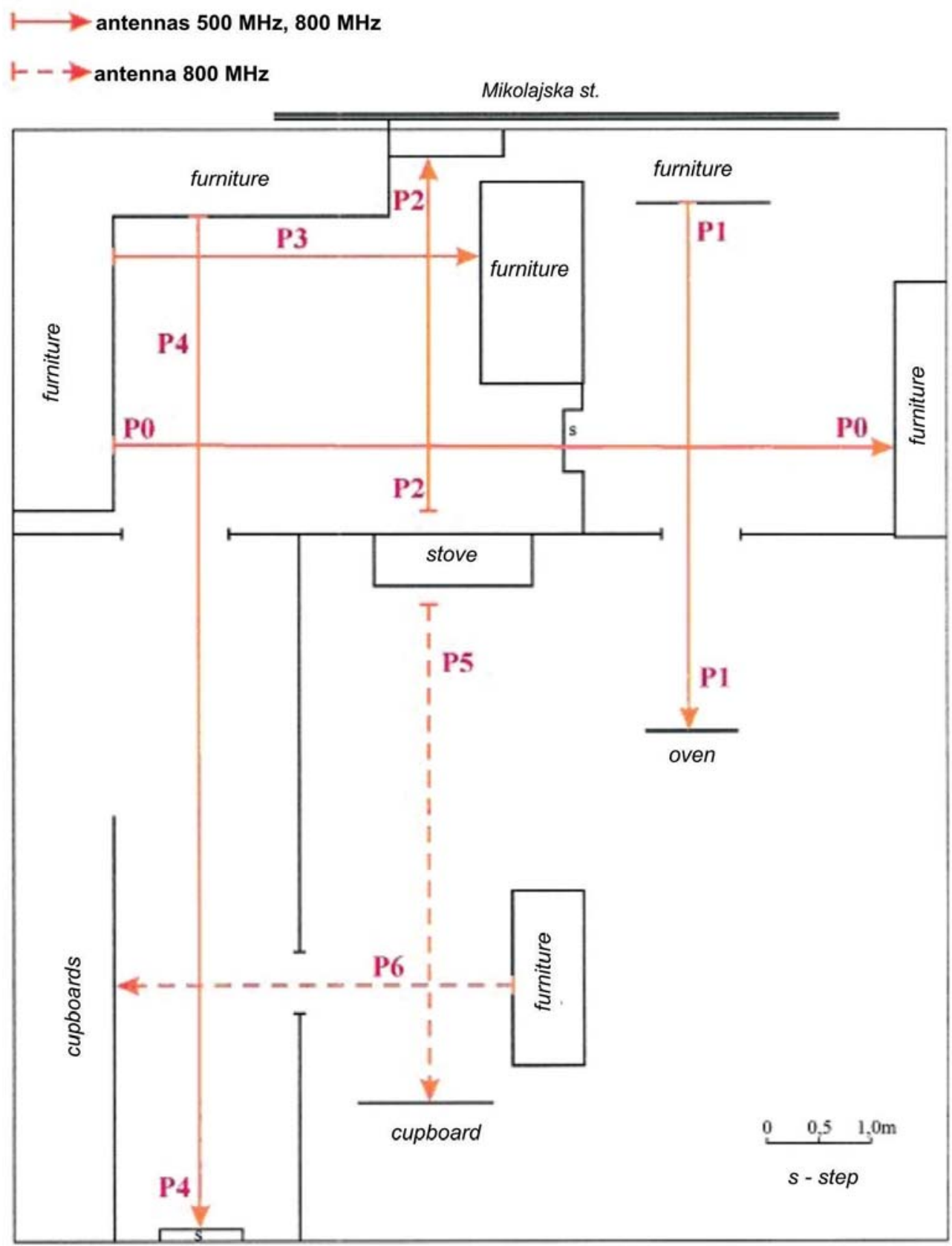

Fig. 1. Location of profiles for the GPR measurements where antennas with frequencies of $500 \mathrm{MHz}$ and $800 \mathrm{MHz}$ were used 


\section{EXPERIMENTAL INSPECTION}

Data from measurements were processed using the Reflex Program. The average wave velocity $v=0.09 \mathrm{~m} / \mathrm{ns}$ was assumed in the studied area, because it was assumed that clay and sand mixture was the dominant material of which the medium is constructed.

The echograms obtained during the measurements were processed with the following procedures:

- move starttime (data were shifted based on the starttime given by manual input);

- background removal (2D procedure, creates a mean trace of the profile and subtracts this trace from each trace);

- subtract-DC-shift (1D procedure, calculates from the given time range a DC-value and subtracts this value from all data values);

- passing frequencies filter: from $250 \mathrm{MHz}$ to $700 \mathrm{MHz}$ for measurements with $500 \mathrm{MHz}$ antenna and from $400 \mathrm{MHz}$ to $1.1 \mathrm{GHz}$ for measurements with $800 \mathrm{MHz}$ antenna;

- gain function amplifying the later reflections, being usually weaker.

A penetration depth of about 2.0 meters with antenna $800 \mathrm{MHz}$ and about 2.5 meters with $500 \mathrm{MHz}$ antenna were achieved.

Processed echograms are visible in Figures 2-5.

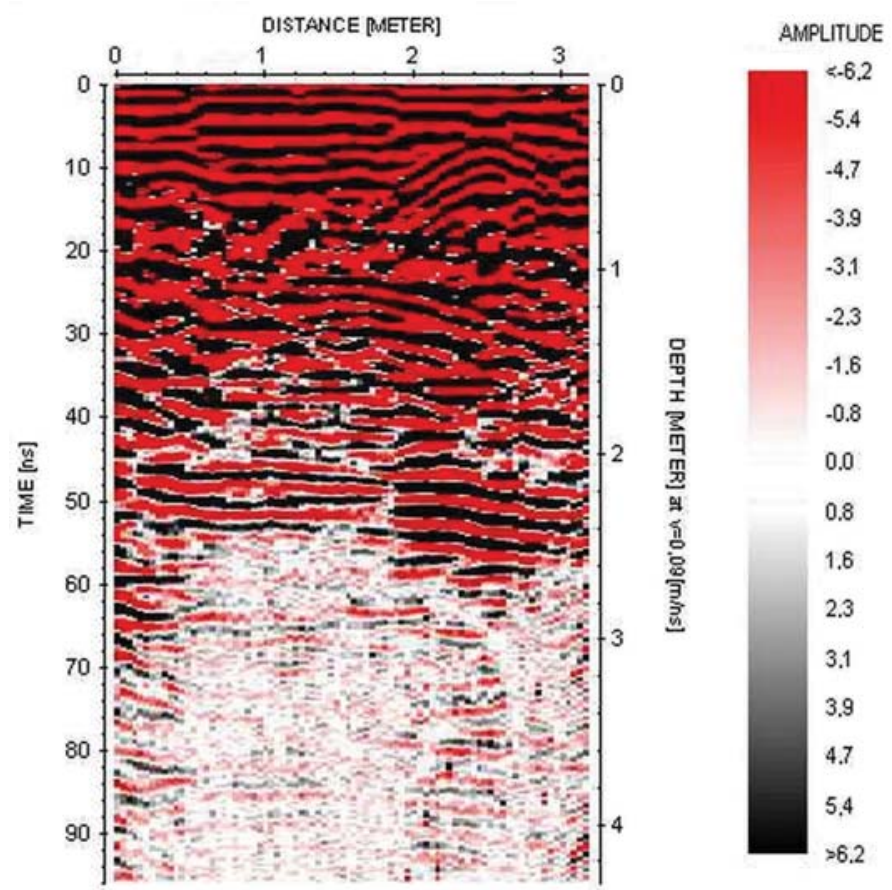

Fig. 2. Processed measurement echogram for profile $\mathrm{P} 3$ obtained by use an antenna with a signal frequency of $500 \mathrm{MHz}$, with amplitude scale attached 


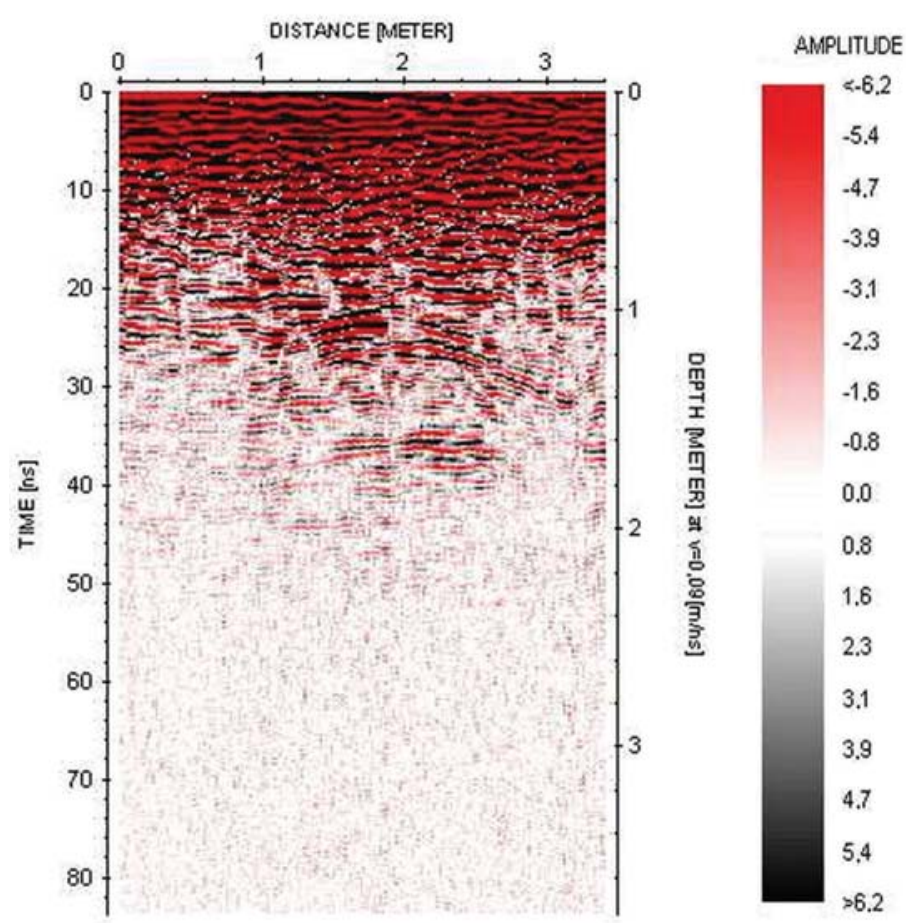

Fig. 3. Processed measurement echogram for profile $\mathrm{P} 3$ obtained by use an antenna with a signal frequency of $800 \mathrm{MHz}$, with amplitude scale attached

One may notice the hyperbola at a depth of about one meter and a distance of about $2 \mathrm{~m}$ on the echograms from profile P3 (Figs 2, 3). Furthermore, the hyperbola is clearly visible at a distance of 2.5 meter of the profile at a depth of about $0.4 \mathrm{~m}$ on echograms obtained with antenna $500 \mathrm{MHz}$.

The door threshold separating the large room from the corridor was marked with white spot (Figs 4, 5).

The wave field recorded on profile P4 can be divided into two parts, with the boundary between them situated about $5 \mathrm{~m}$ from the starting point of the profile. Upon crossing the threshold between the rooms, a brief detachment of the antenna from the ground might have resulted in signal shape disturbance. In the first part of the record (in the large room) the signal has amplitudes much larger than in the corridor. Detailed examination of the echogram shows that the amplitude of the signal after processing, corresponding to depths from $1 \mathrm{~m}$ to $2 \mathrm{~m}$, is much larger in the initial section then in the second part. There are signals with high amplitudes at depth up to $4 \mathrm{~m}$ in the first two meters of the profile. But it is probably caused by reverberation, multiple reflection between the surface of the flat floor and the basement ceiling (Fig. 4). 


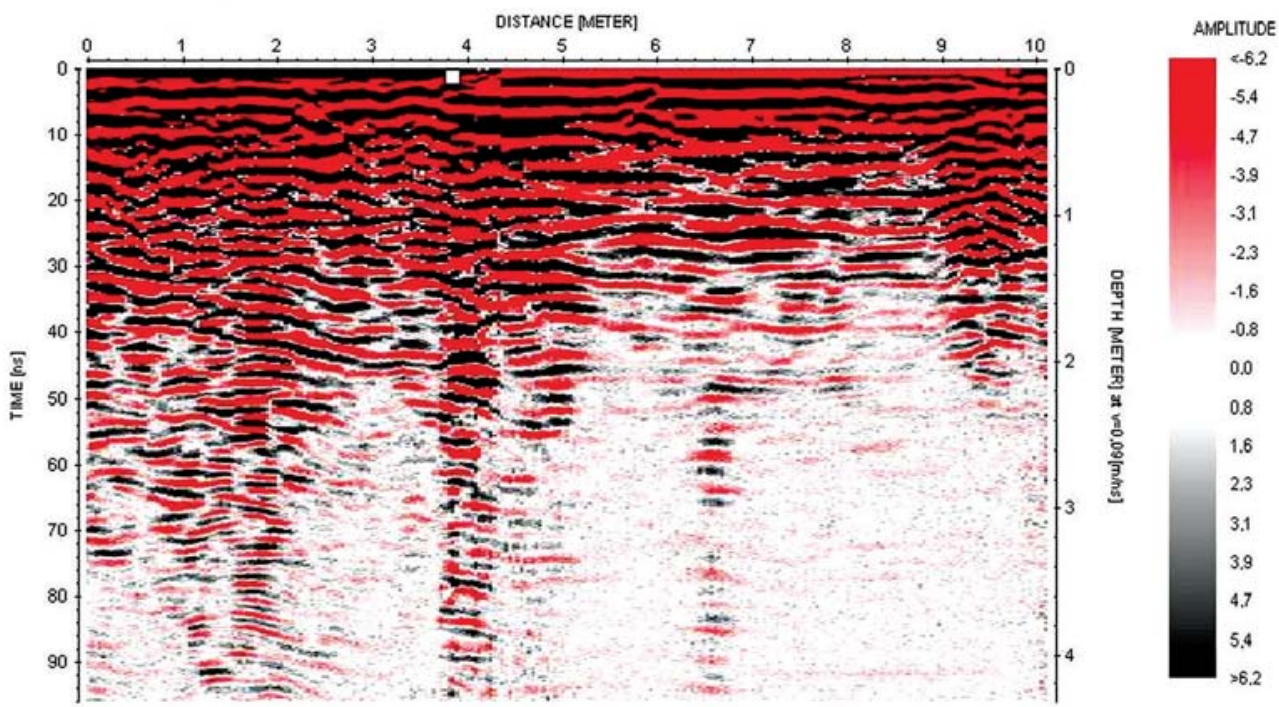

Fig. 4. Processed measurement echogram for profile P4 obtained by use an antenna with a signal frequency of $500 \mathrm{MHz}$, with amplitude scale attached

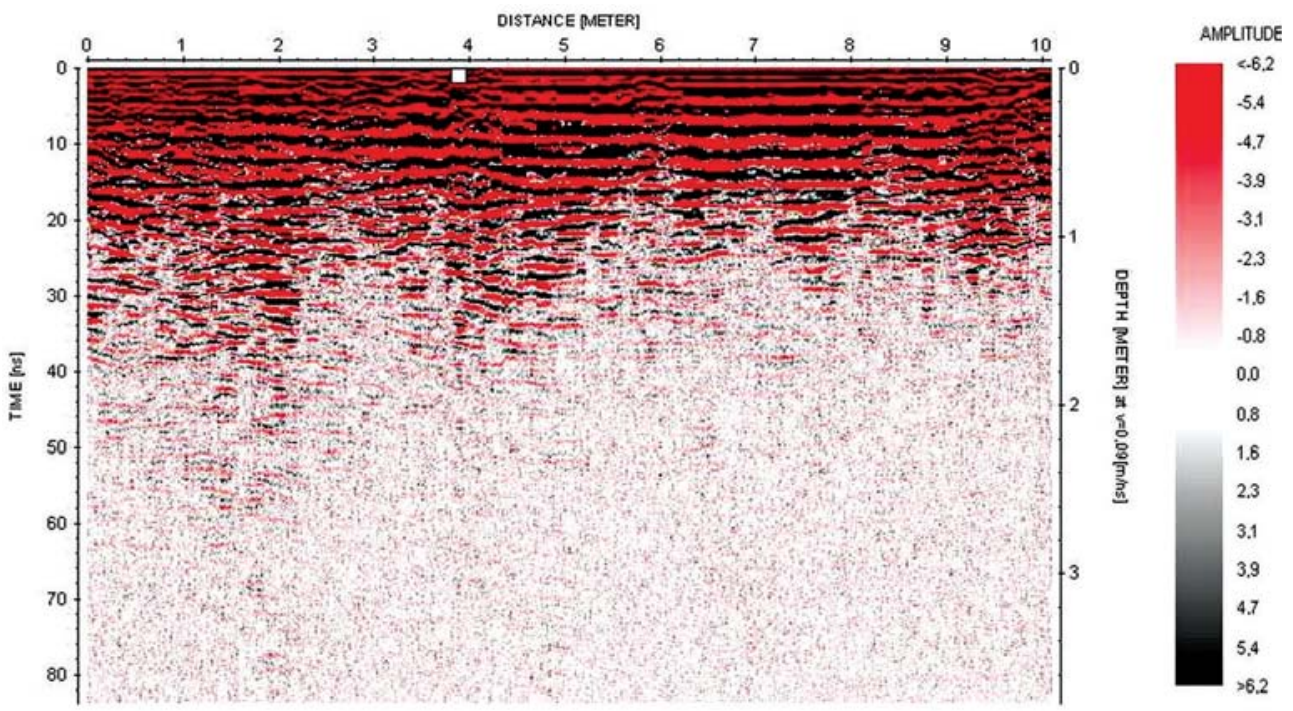

Fig. 5. Processed measurement echogram for profile $\mathrm{P} 4$ obtained by use an antenna with a signal frequency of $800 \mathrm{MHz}$, with amplitude scale attached 
The record's character in the area of the large room may indicate the presence of empty spaces in anomaly zones (a large contrast in dielectric constant on some horizon at a depth of about $1 \mathrm{~m}$ ). Other profiles traversing the large room show similarly high signal amplitudes. The thickness of the cellar ceiling is about one meter. Because the assumed electromagnetic wave velocity may be underestimated, therefore the depth of at which the ceiling actually is located may be smaller. However, the lack of clear wave reflection from the floor of the cellar may indicate that cellar could be partly filled up. The difficulty in interpreting the sign of reflections may be caused by cellar emptiness or due to the basement fill with high-permittivity material. On the other hand a penetration depth is only 2.5 meters and if the basement is deep (more than $3 \mathrm{~m}$ ) the reflection from the bottom will not be registered. What is more, when the signal enters the basement it is already very weak passing through a contrasting boundary between the ceiling and an empty space in the basement. Then it has to reflect from the basement floor and again return through the ceiling, which will weaken it even more.

Also on profile P3 the character of the record, the visible hyperbola at the distance from $1 \mathrm{~m}$ to $2.8 \mathrm{~m}$, at a depth from $1.0 \mathrm{~m}$ to $1.4 \mathrm{~m}$, suggests the existence of a basement under the floor of the room (Figs 2,3). At the end of the profile P4 at a distance ranging from $8.7 \mathrm{~m}$ to $10 \mathrm{~m}$ (near the entrance to the apartment) the character of the record, with high signal amplitudes (at a depths of 0.4-2 m), also indicates the presence of another empty space.

High amplitude is usually observed in the case of investigation of old empty cellars, or basements, filled with clay of high moisture. However, such cases are rare, most probably because a large contrast between the media permittivity values, results in high reflection coefficient. This causes high amplitudes in the registered signals.

Due to the ambiguity of the obtained results, modelling the time evolution of distribution of electromagnetic field was attempted. The adopted model takes into account the existence of voids under a large room and under the entrance to the apartment. Modelling was performed for the two mutually perpendicular profiles $\mathrm{P} 3$ and $\mathrm{P} 4$.

\section{NUMERICAL SIMULATION USING GPRMax}

Modelling of an electromagnetic field in the GPR method has some basic applications. First of all it allows to compare real results of measurement with modelling effects for the assumed construction of the centre. Furthermore, it enables to precise the causes of the anomalies in the recorded echograms (Gołębiowski 2004). In addition, modelling performed for some predefined or predicted medium structure allows for accurate measurement design, including appropriate choice of antennas and the selection of measurement configuration parameters, such as time window and the sampling frequency. Additionally, such modelling enables us to specify the conditions under which the GPR method gives satisfactory results. 
In the modelling it is essential to determine the correct parameters of the medium, especially the distribution of permittivity. As the real construction of the medium is usually complicated, it is a difficult task. An additional difficulty is the nature of the recorded signal, which is different from the signal used in the model. Furthermore, during registration various types of disturbances are recorded (e.g. from the objects on and above the surface). This interference cannot be considered when determining the model parameters (Karczewski 2007).

Modelling of an electromagnetic field consists of solving Maxwell's equations i.e. passage of the impulse in the medium, assuming some model of the medium structure (permittivity and conductivity distribution), some boundary conditions and defined antenna emission. The main methods are described by Sikora (1997).

GPRMax is a shareware program applied for both academic and commercial purposes. It is used to calculate the electromagnetic field distribution at a given $2 \mathrm{D}$ or $3 \mathrm{D}$ model. It was written by Antonis Giannopoulos from the University of Edinburgh in Scotland (Giannopoulos 2005). The program for solving Maxwell's equations uses the FDTD method (Finite Difference Time Domain).

As some restrictions are necessary for the model the following conditions for the $2 \mathrm{D}$ version are assumed:

- medium is linear and isotropic,

- transmitting antenna is the linear source,

- assumed medium parameters (i.e. permittivity) are not frequency dependent.

The input file with data for modelling can be created in any editor in ASCII format. The size of the model, the dimensions of the cell discretization $\Delta x, \Delta y$ and time window $t_{w}$ must be specified in the input file header. The discretization step of the grid depends on the wavelength of the GPR signal. The rule is that it must be at least 10 times smaller than the basic wavelength of the signal.

To receive successfully the solution for the field time evolution in this procedure, the discretization steps values $\Delta x, \Delta y$ and $\Delta t$ cannot be defined independently (Giannopoulo 2005). They are connected with the relationship (for a $2 \mathrm{D}$ model):

$$
\Delta t \leq \frac{1}{c \sqrt{\frac{1}{(\Delta x)^{2}}+\frac{1}{(\Delta y)^{2}}}},
$$

where $c$ - velocity of electromagnetic wave in vacuum.

One of the most difficult problem in modelling of the electromagnetic field evolution applies to open borders. The problem is that the electromagnetic field values in a large area (declared to avoid arrival of boundary reflection to the receiver) cannot be calculated directly by numerical methods such as are used in the model. Therefore an approximation is used by introduction of absorbing boundary condition $(\mathrm{ABC})$ at a suitable distance from the so- 
urce to reduce the amount of calculations. The role of ABC is "to absorb" electromagnetic waves and thus simulate an unlimited space. If the input file does not specify the parameters for the $\mathrm{ABC}$, it will be introduced to the model by default conditions. ABC gives good results (without any significant artificial reflections), if all the sources and objects are placed at a distance of at least 15 discretization cells from the border.

The information following header in the input file concerns the physical parameters in the model medium. The necessary parameters are: dielectric constant, magnetic permeability and conductivity. Properties for ideal conductor (pec) and air (free_space) are programmed by default.

The program allows specifying the anomalous objects and areas of the model - coordinate, shaping, type of objects and the medium from which they are made. A rectangle, a circle and a triangle are possible shapes. Size of model and defined objects should be given in meters. The lower left corner is the beginning of the coordinate system. Other commands apply to identify the type of source (frequency and signal type e.g. Ricker's, Gauss), initial position of antennas, the distance between the transmitting and receiving antenna. It is necessary to determine the way in which antennas move along the profile. The input file determines also: the name of the file defining geometry of the system and format of the output file (ASCII or binary). The results of such modelling can be visualized in Matlab programme using a script attached to the program.

\section{RESULTS AND DISCUSSION}

\section{Profile P3}

The geometry of the model (Fig. 6) was based on the profile P3 echograms obtained using antennas with frequencies of $500 \mathrm{MHz}$ and $800 \mathrm{MHz}$ (Figs 2, 3). The model assumes the existence of a circular conductor of a diameter $0.1 \mathrm{~m}$ at a depth of about $0.4 \mathrm{~m}$ at distance $2.2 \mathrm{~m}$ (diffraction hyperbole corresponding to the object is shown in Figure 2).

The model assumes that the electromagnetic signal is a Ricker's signal with a dominant frequency of $500 \mathrm{MHz}$.

\section{Profile P4}

The geometry of the model (Fig. 7) along the profile P4 was based on echograms obtained with antennas with frequencies of $500 \mathrm{MHz}$ and $800 \mathrm{MHz}$ (Figs 4, 5). The model assumes the presence just under the floor of a conductor, rectangular in cross-section and with dimensions of $0.2 \mathrm{~m}$ to $0.3 \mathrm{~m}$. This assumption facilitates the verification if the signal shape disturbance on the echogram from measurements at $4 \mathrm{~m}$ length is caused by the detachment antenna from the ground or by presence of some metal object.

The model assumes that the broadcast signal is a Ricker's signal with a dominant frequency of $500 \mathrm{MHz}$. 


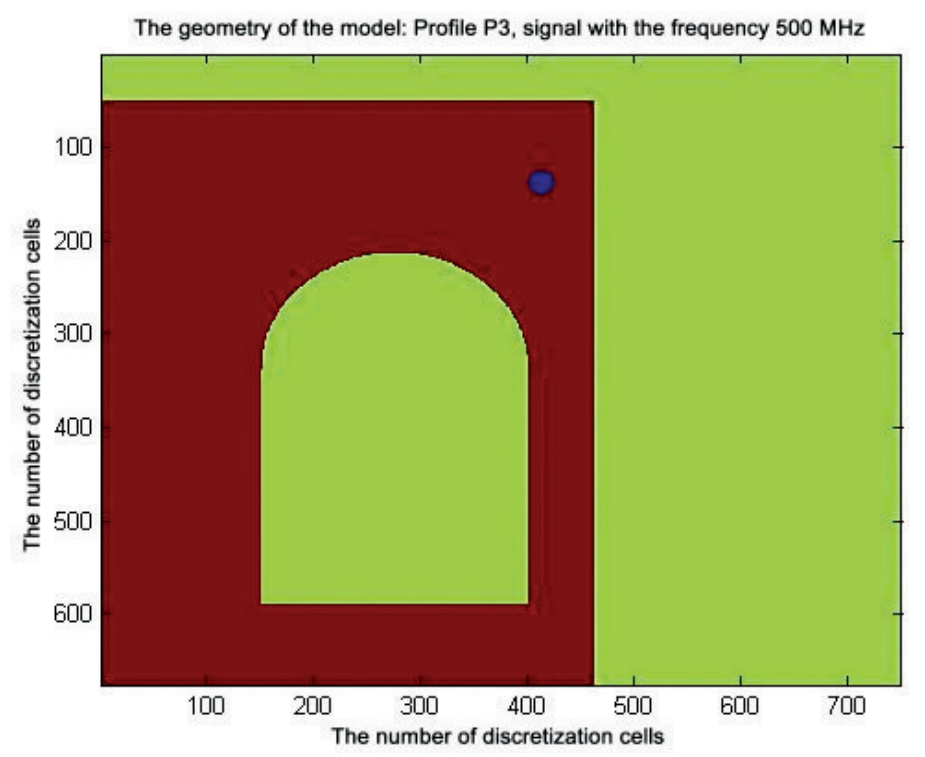

Fig. 6. The geometry of the resulting model of the medium for profile P3, the vertical and horizontal axis gives the number of discretization cells

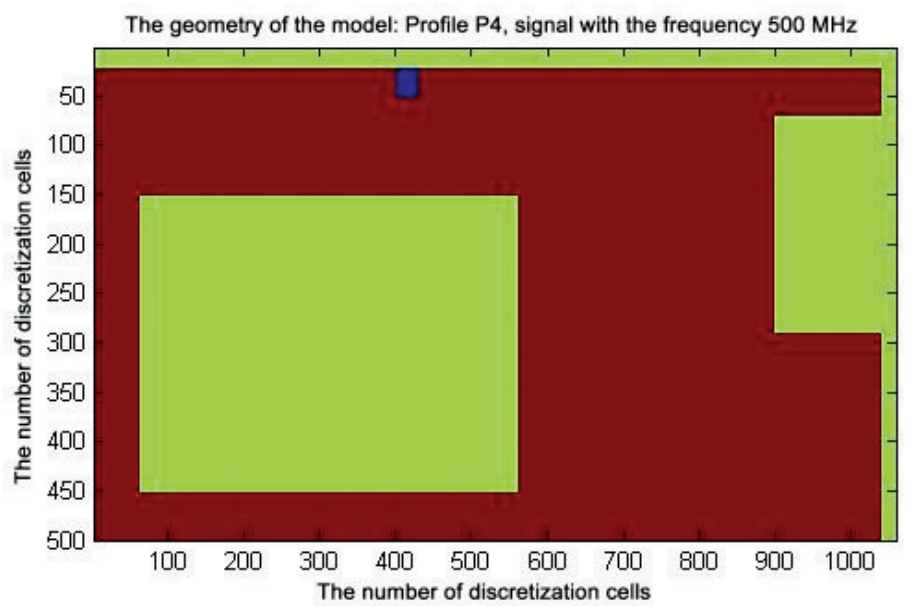

Fig. 7. The geometry of the resulting model of the medium for profile P4, the vertical and horizontal axis gives the number of discretization cells

\section{Discussion}

Echograms resulting from modelling and processed in Reflex program by applying procedures background removal, subtracting average, DC-shift and gain function are presented below. Processing without background removal and subtracting average procedures was also undertaken because they may remove horizontal reflections. 
Modelling for profile P3 gave echogram comparable to the record obtained during measurements. There is a clear hyperbola coming from the arch-shape basement ceiling at a depth of approximately one meter (Fig. 8). Also a reflection at depth of about 0.4 meters (which is derived from the object of high conductivity or high permittivity material in the model) is similar to the reflex on echogram from measurements.

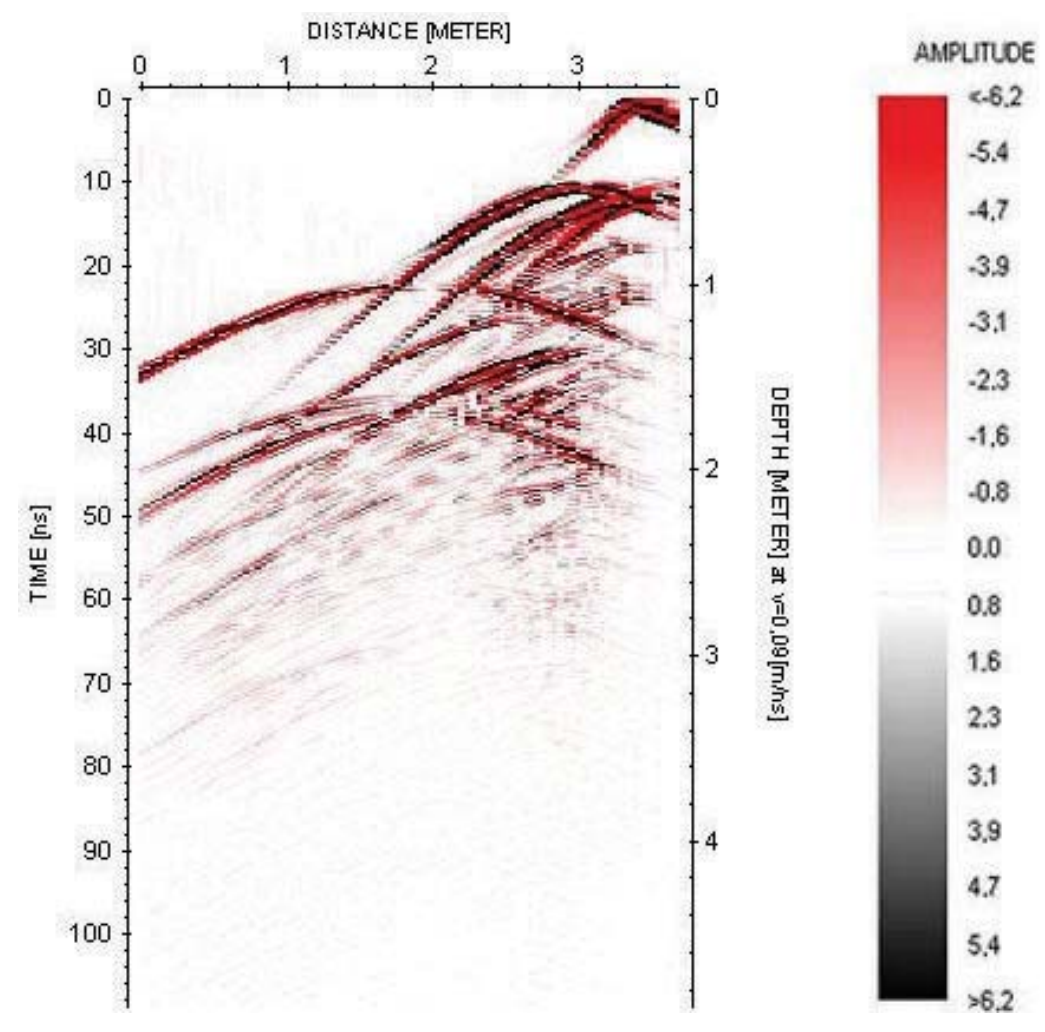

Fig. 8. The modelling results echogram for profile $\mathrm{P} 3$, dominant signal frequency of $500 \mathrm{MHz}$ with amplitude scale attached

There is no clear reflection from the bottom of the basement on the modelling echogram. Since the velocity of electromagnetic wave in the air is much higher than in any other medium so on the echogram reflection from the basement floor should be "less deep" than follows from assumed geometry.

The result of the profile P4 modelling gives results that are not entirely comparable with the measurement echogram. Although at the end of the profile, at the distance of about $9 \mathrm{~m}$ (Figs 4, 9), the reflections of large amplitudes are visible on both echograms. It may be inferred that the assumed model for this part of the profile is correct and in fact there is 
not a completely buried space below the floor. Moreover very strong reflection with long hyperbola arms at $4 \mathrm{~m}$ length of the profile just under the surface is due to presence of metal object (Fig. 9). This is visible only on the echogram from modelling and means that signal shape disturbance is caused by the detachment antenna from the ground. However, in the initial part of the profile P4 (the fifth meters long) reflections with high amplitude also appear at depths $1.3 \mathrm{~m}$. Hyperbolas marked with green arrows in figure 9 are the result of wave diffraction on the upper corners of the rectangle (imaging the ceiling of the empty basement in the assumed model). However, there is no clear hyperbole here in the measurement echogram. Such an image suggests that the room below the floor could be a basement but with a different shape of ceiling than assumed in the model. Therefore, modelling was carried out assuming different geometry of the medium (Fig. 10).

In this case the signal was set with the frequency of $200 \mathrm{MHz}$. The aim was to check whether the lower frequency, and thus a longer wavelength, manages to get a clear reflection from the floor of the basement. The hyperbola visible in the resulting echogram (Fig. 11) on about $22 \mathrm{~ns}$ coincides with the arc-shaped reflection that could be interpreted as hyperbola from echogram from measurements. It follows that the ceiling of the basement would be dome-shaped. Despite using a lower frequency signal no clear reflection from the basement floor on the echogram is observed. Only multi reflections are visible, even after applying gain function.

Modelling was also carried out for the profile P3 for a basement partially buried by wet sand (Fig. 12). The modelling assumed a signal with a dominant frequency of $500 \mathrm{MHz}$.

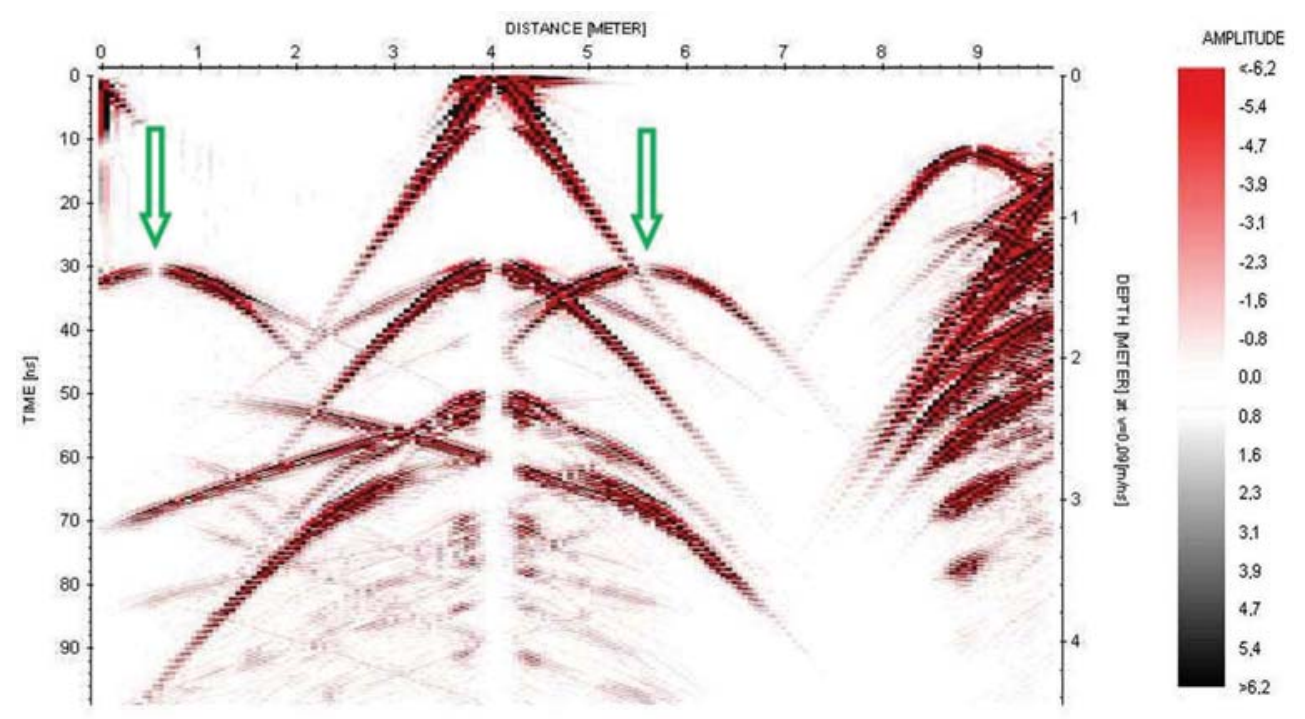

Fig. 9. The modelling results echogram for profile $\mathrm{P} 4$, dominant signal frequency of $500 \mathrm{MHz}$ with amplitude scale attached 


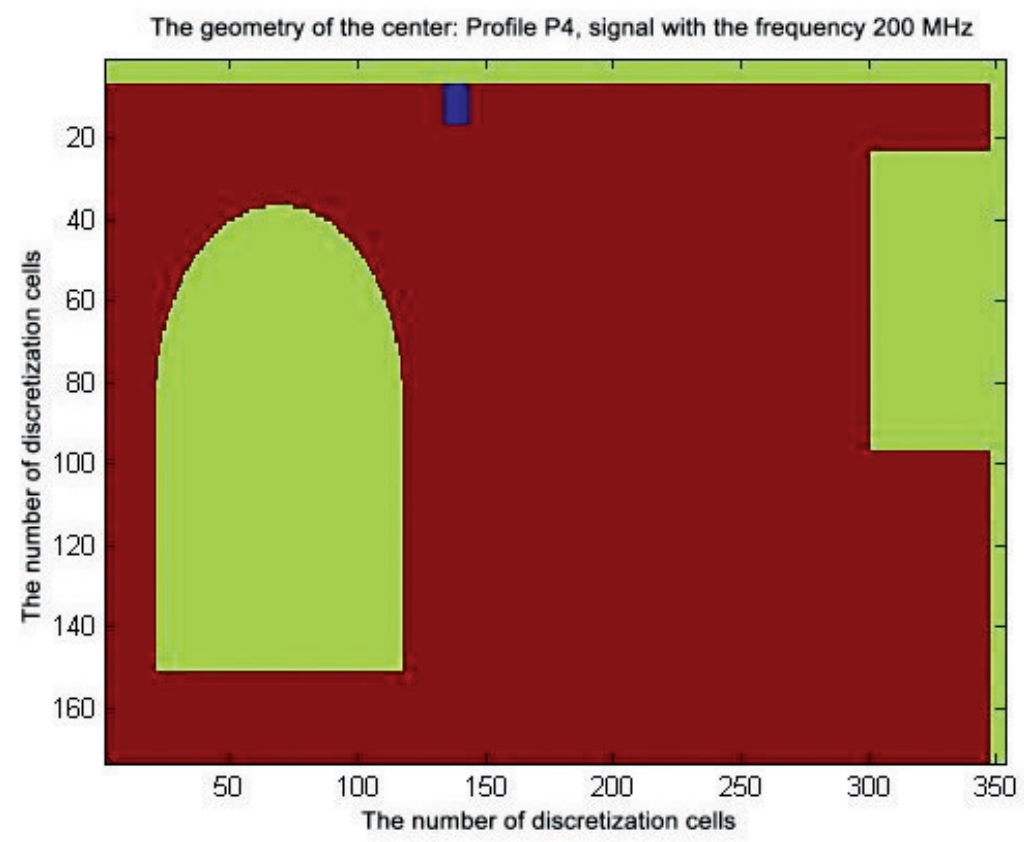

Fig. 10. The geometry of the assumed model of the medium for profile P4 the shape of the basement ceiling is changed

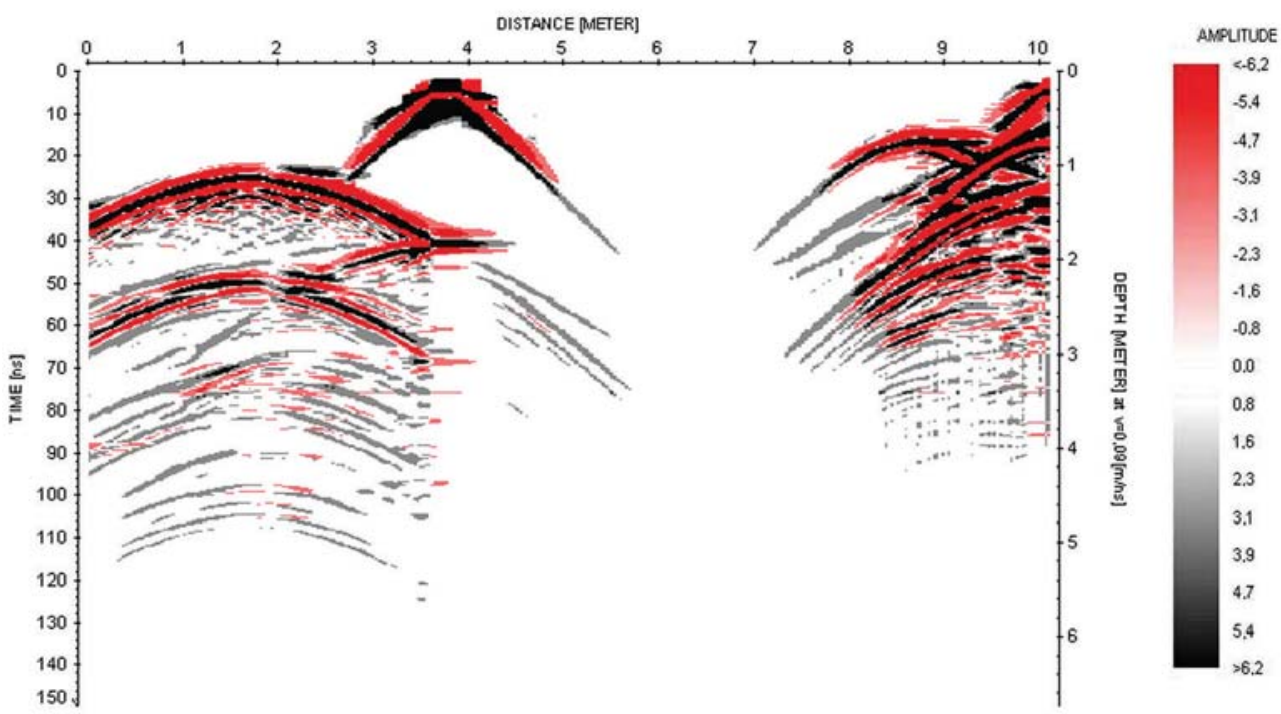

Fig. 11. The modelling results echogram for profile P4 - arch shape basement ceiling, dominant signal frequency of $500 \mathrm{MHz}$ with amplitude scale attached 


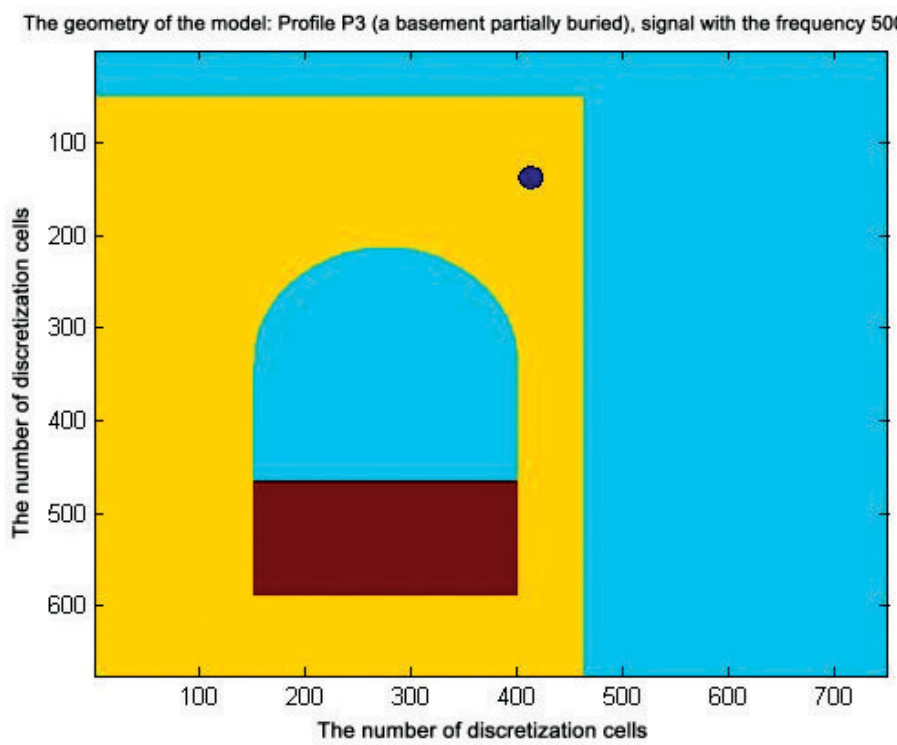

Fig. 12. The geometry of the assumed model of the medium for profile P3 basement partially buried

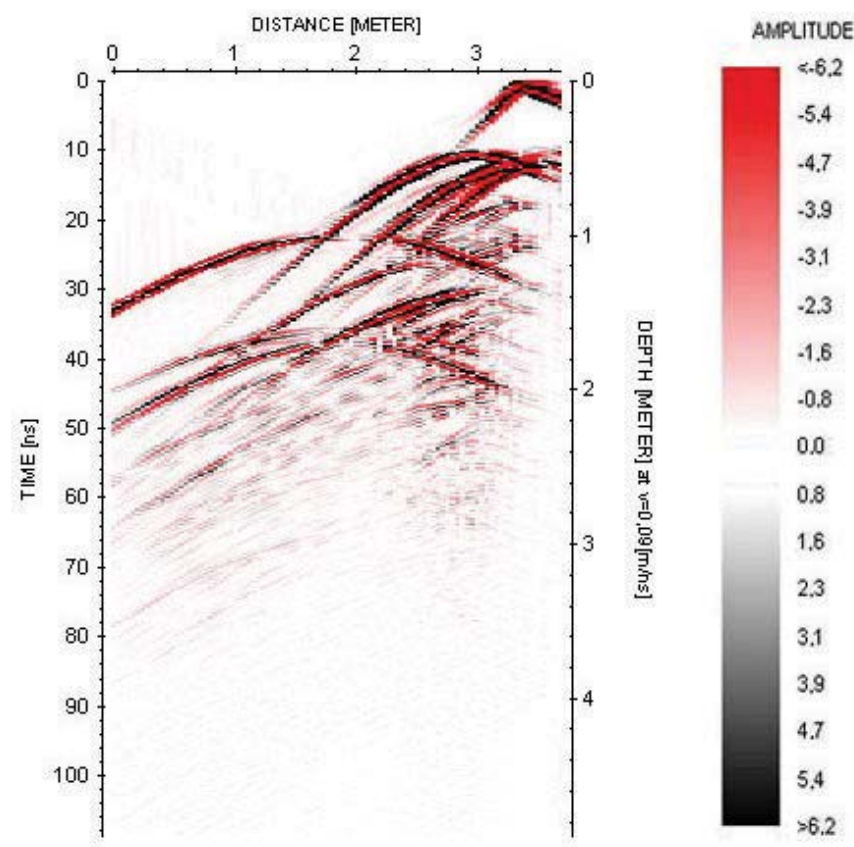

Fig. 13. The modelling results echogram for profile P3 - basement partially filled, dominant signal frequency of $500 \mathrm{MHz}$ with amplitude scale attached 
The received echogram (Fig. 13) is similar to the echogram from modelling for an empty basement (Fig. 8). Even the boundary between the air and moist sand filling the basement at the bottom is not clearly visible even after applying gain function. No reflections from that boundary may be the result of interference of waves reflected from the boundaries between media or assumptions in the model construction.

\section{CONCLUSIONS}

Based on the results of measurements in the investigated tenement it may be concluded that the GPR method was useful in the study of historic residential buildings and applies when looking for voids (such as basements to which direct access is not possible). What is more, this method, due to its high resolution, allows users to locate sometimes objects of small size.

Conducting proper modelling requires the assumption of appropriate distribution of electrical parameters of the medium. The numerical modelling allows us to select the most probable variant interpretation of the resulting data. This is particularly important when there is limited access to the investigation area or the recording of the wave obtained during measurements is ambiguous.

This work confirmed the existence of the basement under the floor of the apartment. Based on echograms obtained from the modelling and processed echograms from measurement, similar reflections were found at a depth of approximately one meter, which is most likely the result of an electromagnetic wave reflection from the basement ceiling. However, reflections from the floor of the basement are not observed on both measured and modelled echograms. For this reason, it was impossible to determine the exact height of the basement after the analysis of the measurement echograms. By comparing the modelling results for the assumed different geometry of the medium the shape and dimensions of the basement ceiling may be specified approximately.

\section{REFERENCES}

Annan A.P., 1999. Practical Processing of GPR Data. Sensors \& Software, [on-line:] http:// www.sensoft.ca.

Annan A.P., 2002. GPR - History, Trends, and Future Developments. Subsurface Sensing Technologies and Applications, 3, 4, 253-270.

Benedetto A., Pensa S., 2007. Indirect diagnosis of pavement structural damages using surface GPR reflection techniques. Journal of Applied Geophysics, 62, 107-123.

Benedetto A., Benedetto F., Tosti F., 2012a. GPR applications for geotechnical stability of transportation infrastructures. Nondestructive Testing and Evaluation, 27, 3, 253-262.

Benedetto A., Manacorda G., Simi A., Tosti F., 2012b. Novel perspectives in bridges inspection using GPR. Nondestructive Testing and Evaluation, 27, 3, 239-251. 
Czaja K., 2010. Zastosowanie metody GPR w badaniach budynków mieszkalnych. Biblioteka Wydziału Geologii, Geofizyki i Ochrony Środowiska AGH, Kraków [M.Sc. Thesis].

Daniels D., 2004. Ground Penetrating Radar. $2^{\text {nd }}$ Edition. The Institution of Electrical Engineers, London.

Giannopoulos A., 2005. GPRMax v.2.0 User's Manual. University of Edinburgh, Scotland.

Gołębiowski T., 2004. Wprowadzenie do metodyki interpretacji badań georadarowych przy użyciu procedury modelowania numerycznego. Przeglad Geologiczny, 52, 7, 563-568.

Grote K., Hubbard S., Rubin Y., 2003. Field-scale estimation of volumetric water content using GPR groundwave techniques. Water Resources Research, 39, 11, DOI: 10.1029/2003 WR002045.

Karczewski J., 2007. Zarys metody georadarowej. UWND AGH, Kraków.

Saarenketo T., Scullion T., 2000. Road evaluation with ground penetrating radar. Journal of Applied Geophysics, 43, 119-138.

Sikora R., 1997. Teoria pola elektromagnetycznego. Wydawnictwa Naukowo-Techniczne, Warszawa. 